\title{
Relevance of Basilar Artery Study in Patients with Subclavian Steal Phenomenon
}

\author{
Maria Jose Garcia-Antelo*, ${ }^{*}$, Alfredo Puy-Nuñez ${ }^{2}$, Oscar Ayo-Martin ${ }^{3}$ and Tomas Segura ${ }^{3}$ \\ ${ }^{1}$ Hospital Universitario A Coruña, A Coruña, Spain \\ ${ }^{2}$ Complejo Hospitalario de Pontevedra, Pontevedra, Spain \\ ${ }^{3}$ Hospital Universitario de Albacete, Albacete, Spain
}

\begin{abstract}
A 72-year-old male presented to the emergency department with gait instability and unclear speech. Computed tomography of the brain showed old lacunar infarcts in basal ganglia. Transcranial Doppler (TCD) sonography was normal. Extracranial Duplex sonography showed indirect hemodynamic signs of bilateral subclavian artery stenosis and both vertebral arteries also showed delayed systolic flow increase. A bilateral subclavian steal phenomenon was suspected, and arm compression tests was performed. The tests promoted reverse flow in the right VA, loss of diastolic flow in the left VA and interestingly, the normal anterograde BA flow became retrograde. Although subclavian steal is likely to be an innocuous phenomenon for the majority of our patients, it is probable that the presence of a hemodynamic effect on the basilar artery may identify those who are at special risk of neurologic symptoms. So, we recommend TCD study in all patients suffering SSP to rule out the possibility of a BA steal phenomenon.
\end{abstract}

Keywords: Subclavian steal, basilar steal, cerebrovascular disease, transcranial doppler ultrasound, vertebro-basilar symptoms.

\section{INTRODUCTION}

The subclavian steal phenomenon (SSP) is characterized by retrograde flow within a vertebral artery (VA) ipsilateral to an upstream proximal high-grade subclavian artery (SA) stenosis or occlusion. Three grades of SSP have been reported [1], but this classification only takes the ipsilateral VA flow characteristics into account.

The SSP may additionally cause a basilar artery (BA) steal phenomenon (BSP). When this occurs, the inversion of flow in the subclavian artery provokes additional retrograde flow in the basilar artery. Most of these patients are neurologically symptomatic [2]; therefore, transcranial Doppler ultrasound is essential during the early diagnosis.

We report a case of a patient with stroke because of bilateral SSP and BSP.

\section{CASE REPORT}

A 72-year-old male, smoker with a past medical history of arterial hypertension, high blood cholesterol and ischemic cardiomyopathy, had suffered a transient episode of slurred speech a week earlier that resolved within 4 hours.

The patient presented to the emergency department with gait instability and unclear speech. Physical examination of the patient revealed blood pressure 130/60 on the right arm and $150 / 75$ on the left, weak pulse in both legs and no radial pulse in the arms. Neurological examination showed unclear speech and gait instability without other abnormalities.

*Address correspondence to this author at the Hospital Universitario A Coruña. As Xubias, 84, 15006, A Coruña, A Coruña, Spain;

Tel: 657091302; Fax: 034981178001, 034981178163;

E-mail: marigaran@yahoo.es
The National Institute of Health Stroke Scale (NIHSS) was 2. The Canadian scale was 10.

Electrocardiogram revealed sinus rhythm. Computed tomography (CT) of the brain showed prior lacunar infarcts in both basal ganglia and a subacute lacunar infarction affecting left paramedian thalamus.

Transcranial Doppler ultrasound was normal. Extracranial Duplex ultrasound showed diffuse cervical atheromatosis including non-relevant $(<50 \%)$ carotid plaques and indirect hemodynamic signs of bilateral subclavian artery (SA) stenosis (delayed increase in systolic flow with monophasic flow profile instead of a normal triphasic profile). Interestingly, both vertebral arteries (VA) also showed delayed systolic flow increase (Fig. 1). A bilateral subclavian steal phenomenon was then suspected and arm compression test with a blood-pressure cuff was performed. The test was repeated on both arms; release of the pressure cuff led to reverse flow in the right VA and loss of diastolic flow in the left VA (Fig. 1). The arm compression test was repeated because of the patient's neurological symptoms while we recorded basilar artery (BA) waveforms (Fig. 2) by means of transcranial Doppler. The normal anterograde BA flow became retrograde following the causative ischemia test in either arm (Fig. 2).

The suspected diagnosis was athero-embolic stroke affecting left thalamus due to proximal left subclavian atheromatosis. Additionally, the patient suffered bilateral subclavian steal phenomenon, mainly on the right side. The patient was admitted to the stroke unit. A CT angiography performed 24 hours later confirmed the data from the ultrasound study. It showed stenosis about $77 \%$ of the right subclavian artery and $50 \%$ of the left. 


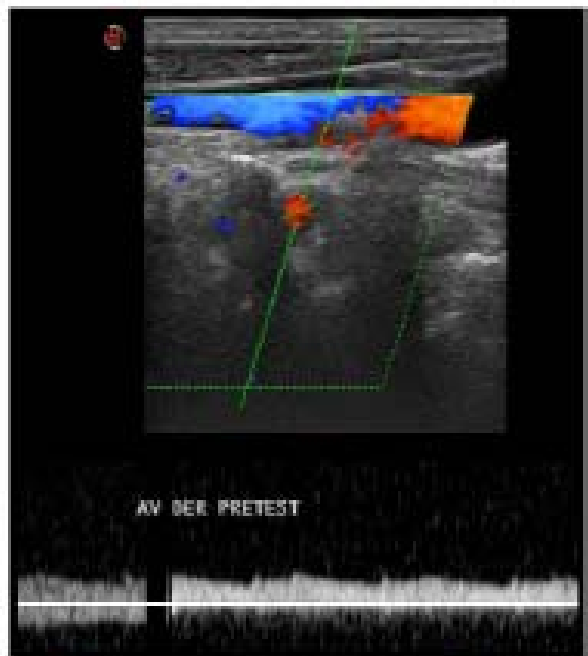

a

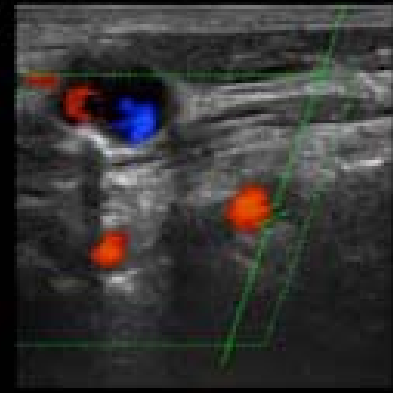

AV tap partert

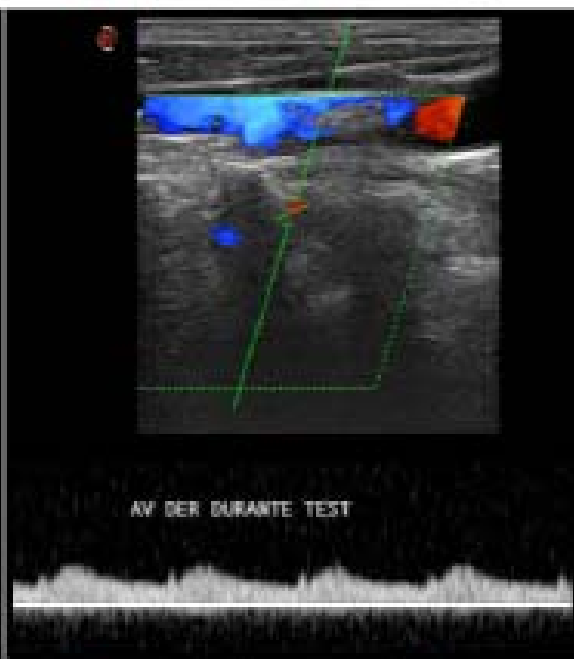

(1.)

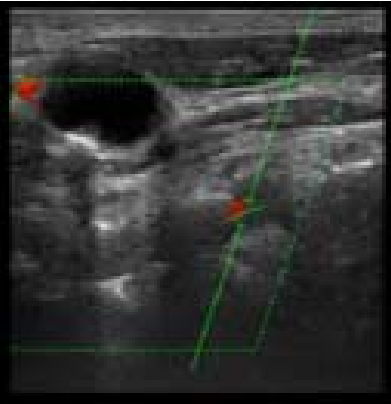

AV 190 TMIEATES

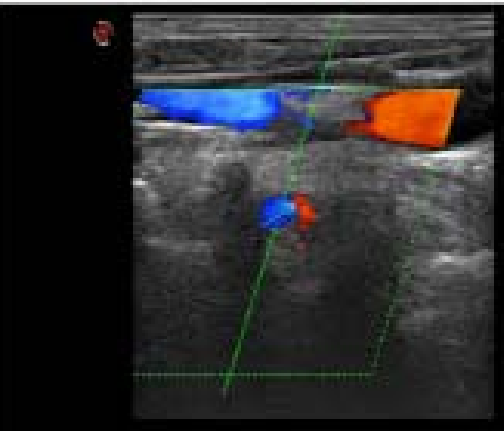

aV DER POST TEST

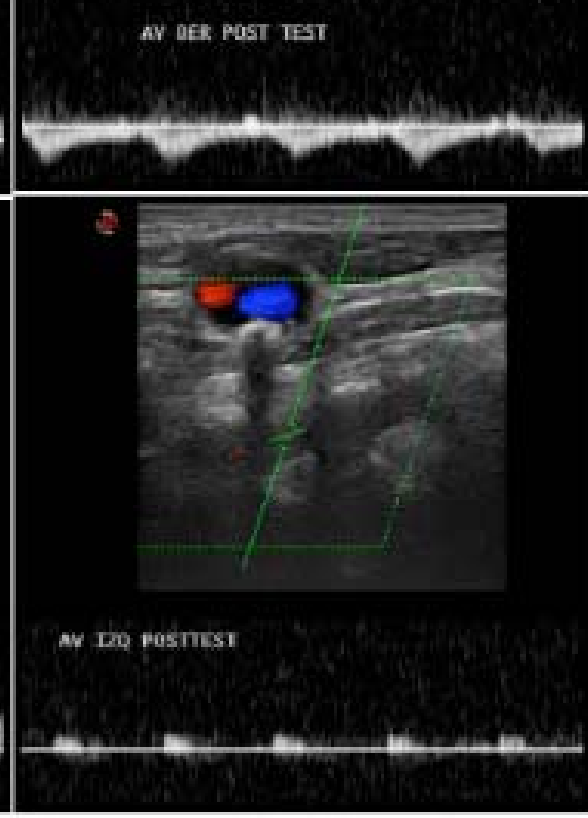

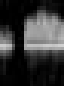

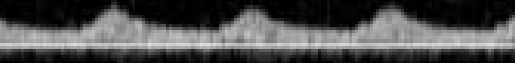

Fig. (1). Extracranial color Duplex study of vertebral arteries before (left side), during (middle position), and upon completion (right position) of the arm compression test. Right VA (upper images) reveals initial anterograde flow that became retrograde after cuff release. Left VA (lower images) also shows initial anterograde flow with loss of diastolic flow at the end of the test.

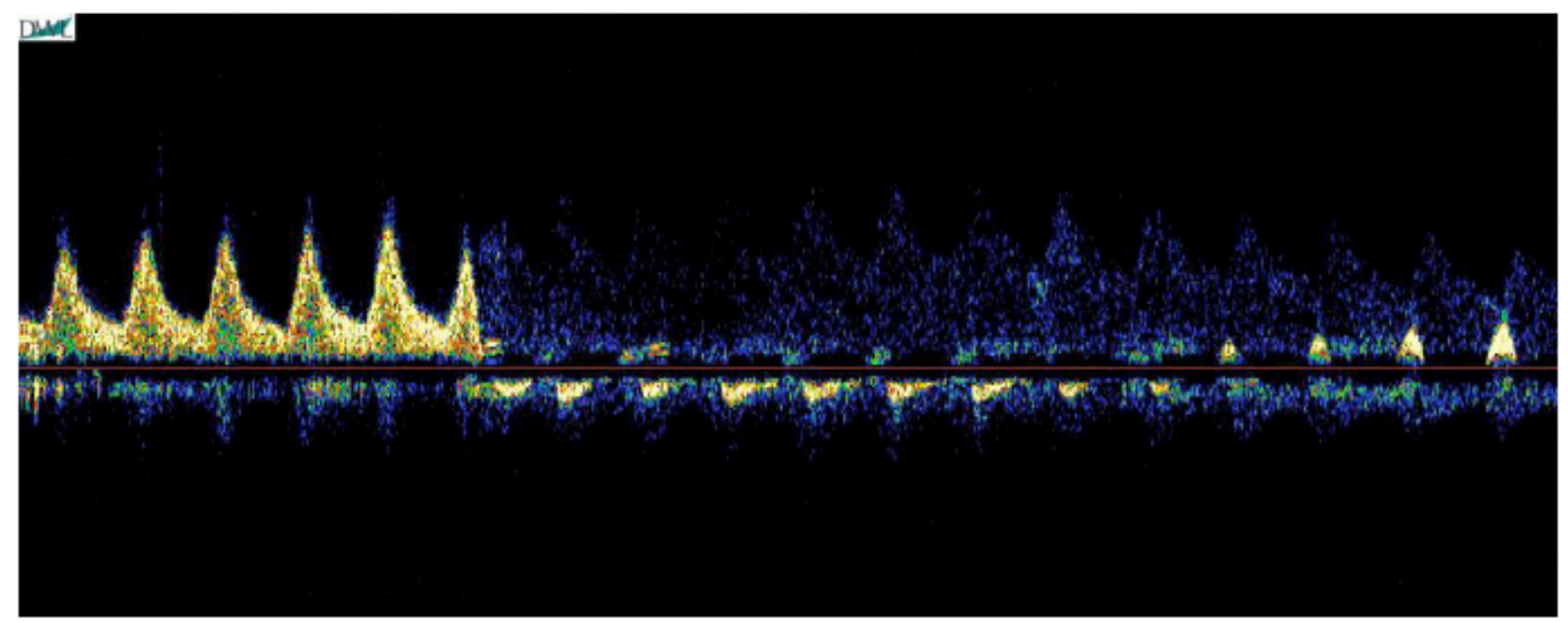

Fig. (2). Basilar Steal Phenomenon. Normal basilar flow converted to retrograde flow upon release of the blood-pressure cuff. Basilar flow is initially anterograde. Following the ischemia test, the basilar artery shows minimal and retrograde flow. 
Stenting was performed on both subclavian arteries in the same procedure free of complications. Pharmacological double anti-aggregation treatment was established; acetylsalicylic acid $150 \mathrm{mg} / \mathrm{d}$ and clopidogrel $75 \mathrm{mg} / \mathrm{d}$.

After two months, the patient was asymptomatic. Cervical ultrasonography confirmed permeability of stents in the subclavian arteries and absence of steal phenomenon.

\section{DISCUSSION}

Harrison (1829) was the first to realize the impact of subclavian stenosis in the vertebro-basilar system. Contorni (1960) used angiography to demonstrate retrograde flow in the ipsilateral vertebral artery proximal to a subclavian stenosis in an asymptomatic patient. However, Fisher (1961) was the first to refer to this phenomenon by its current name: subclavian steal syndrome.

Subclavian steal phenomenon is characterized by a reversal of flow within a vertebral artery ipsilateral to an upstream proximal high-grade subclavian artery stenosis or occlusion. Most patients are asymptomatic; however, symptoms in the vertebro-basilar territory may occur. Typical symptoms are episodic dizziness, diplopia, dysartria, perioral numbness, visual disturbances, drop attacks, tinnitus and nausea [3]

Three grades of SSP have been reported [1], but this classification only takes into account the ipsilateral VA flow characteristics. Grade 0: no subclavian steal. Grade 1: systolic deceleration in the VA. Grade 2: alternating flow in the VA. Grade 3: reversed flow in the VA. The SSP may additionally provoke a basilar artery (BA) steal phenomenon (BSP), and most of these patients are neurologically symptomatic. As such, a new classification taking into account the flow of the basilar artery is probably necessary.

De Bray et al. (1994) reviewed a series of 55 patients with permanent or intermittent subclavian steal syndrome and found that 8 patients had defined vertebro-basilar symptoms. In 7 of these 8 cases, a basilar steal phenomenon occurred. This study concludes that in the subgroup of patients with basilar steal phenomenon, the risk of stroke was greater, as in our case. It is essential to demonstrate that reverse flow of the basilar artery exists; ischemia tests should be performed if necessary.

\section{CONCLUSION}

Although subclavian steal is likely to be an innocuous phenomenon for the majority of our patients, it is probable that the presence of a hemodynamic effect on the basilar artery may identify those at special risk of neurological symptoms. Therefore, we recommend TCD study in all patients suffering SSP to rule out the possibility of a BA steal phenomenon.

\section{CLARIFICATION}

I think somewhere in the article should be noted that this work was presented in part at the XIX European Stroke Conference (May 2010) in Barcelona, Spain.

\section{REFERENCES}

[1] Thomassen L, Aarli JA. Subclavian steal phenomenon. Clinical and hemodynamic aspects. Acta Neurologica Scandinavica 1994; 90: 241-244.

[2] De Bray JM, et al. Effect of subclavian syndrome on the basilar artery. Acta Neurologica Scandinavica 1994; 90: 174-178.

[3] Kizilkilic O, Oguzkurt L, Tercan F, et al. Subclavian steal syndrome from the ipsilateral vertebral artery. Am J Neuroradiol 2004; 25: 1089-1091. 